\title{
Calibration of Total Pressure Gauges in the UHV and XHV Regions
}

\author{
Karl JOUSTEN \\ Phys.-Techn. Bundesanstalt, Abbestr. 2-12, D-10587 Berlin
}

(Received April 20, 1994, Accepted July 2, 1994)

\begin{abstract}
When total pressures decrease to the XHV region, measurements with ionization gauges become increasingly sensitive to disturbances such as electron- or photonstimulated desorption, outgassing of the gauges, and production of secondary electrons by X-rays. Therefore, it is very important to have a reliable pressure scale also in the XHV region. The laboratory for vacuum physics at the Physikalisch-Technische Bundesanstalt (PTB) in Germany provides a calibration system with molecular beam expansion for pressures down to $10^{-10} \mathrm{~Pa}$ and is building a new calibration system based on continuous expansion for a lower calibration limit in the $10^{-11} \mathrm{~Pa}$ decade. The advantages and disadvantages of the two systems will be discussed and calibrations with the molecular beam system will be presented. For determining the outgassing rate of the vacuum chamber of the new calibration system, a new method was applied and compared to the classical methods, the throughput method and the pressure rise method. In particular it was checked whether the outgassing rate depends on the pumping speed applied to the chamber as proposed in a theory by Malev.
\end{abstract}

\section{Introduction}

The principle of all methods of realizing the pressure scale in the vacuum regime is to measure the test gas at a higher level of pressure and then to expand it in a well-defined manner to a lower level. Although other principles can be conceived, e.g., ionization of all particles in a certain volume and measurement of their charge, only the above-mentioned principle is established today at the metrological institutes for calibrating vacuum gauges.

The kind of expansion used depends on the vacuum regime. While static expansion is used in fine and high vacuum, continuous expansion is used in high and ultrahigh vacuum (UHV), and molecular beam expansion, finally, is used in ultrahigh and extremelyhigh vacuum (XHV).

The principle and practice at PTB of molecular beam expansion to calibrate gauges in low UHV and in XHV will be described in the following section. In principle, it is also possible to expand the continuous expansion into the XHV range. This approach is attempted in a new continuous expansion standard built at PTB, which will be described in section 3. For measuring the low required outgass- ing rate in this system, a new method of outgassing rate measurement was developed, which also easily allows testing whether the outgassing rate depends on the pumping speed applied to the chamber as proposed in a theory of Malev. ${ }^{1)}$ This will be described in section 4 .

\section{Calibration in the XHV Range with a Molecu- lar Beam Expansion Standard}

The main parts of the molecular beam expansion standard at PTB are the following: a chamber (here called the Knudsen cell) with a thin orifice where the molecular beam effuses out, a calibration chamber with another orifice as the entrance for the molecular beam, and a cryopanel $(4.2 \mathrm{~K})$ in between.

The principle of operation is as follows. By a leak valve, the desired pressure $p_{\mathrm{K}}$ in the Knudsen cell is adjusted. This pressure is measured with a calibrated ion gauge (reference gauge). The molecular beam effusing out of the cell is mostly pumped away by the cryogenic pump. A small fraction of the molecular beam, however, hits the entrance orifice of the calibration chamber. In equilibrium, the number of particles entering the calibration 


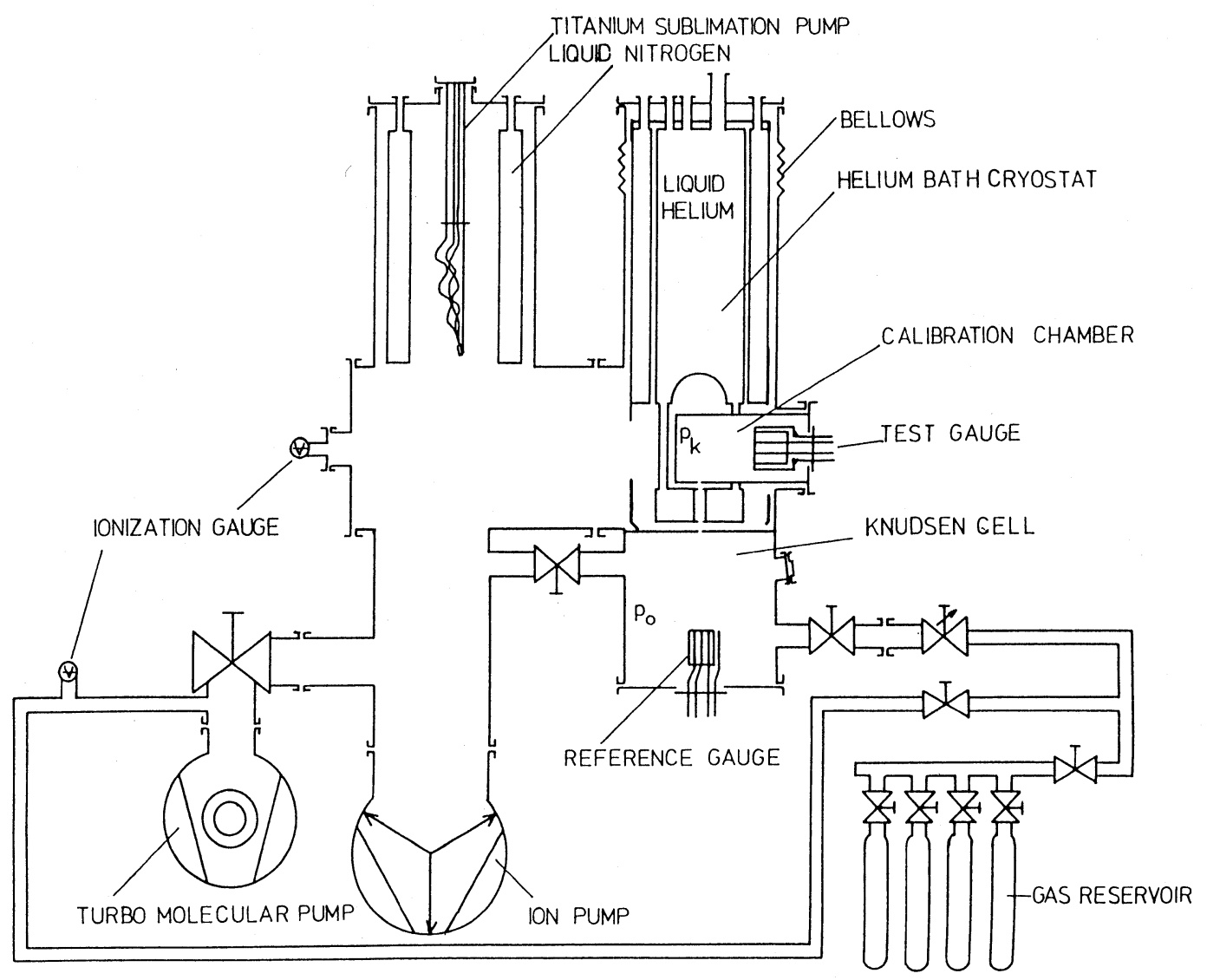

Fig. 1 Scheme of the molecular beam expansion system.

chamber will be equal to the number of particles leaving it; a constant pressure $p_{\text {cal }}$ is established. The ratio $f=p_{\mathrm{K}} / p_{\text {cal }}$ is independent of pressure in the molecular regime. Ideally, $f$ may be calculated from the geometry of the molecular beam. At PTB the ratio was measured by two calibrated ionization gauges, since one of the assumptions for calculating $f$ is that the two chambers are at equal or at least well-defined temperatures, which could not be fulfilled due to the immediate neighbourhood of the cryosystem.

$f$ was measured as 7300 with a total uncertainty of $1.5 \%$. No long-term instability was found. This means that for $p_{\text {cal }}=1 \times 10^{-10} \mathrm{~Pa}, p_{\mathrm{K}}=7.3 \times 10^{-7}$ $\mathrm{Pa}$ must be adjusted. $f$ was measured between $10^{-6}$ $\mathrm{Pa}$ and $10^{-3} \mathrm{~Pa}$ for $p_{\mathrm{K}}$. The standard is used for calibrations between $10^{-10} \mathrm{~Pa}$ and $10^{-6} \mathrm{~Pa}$.

The total uncertainty of $p_{\text {cal }}$ is simply given by

$$
\frac{\Delta p_{\text {cal }}}{P_{\text {cal }}}=\frac{\Delta p_{\mathrm{K}}}{p_{\mathrm{K}}}+\frac{\Delta f}{f}
$$

The uncertainty of $p_{\mathrm{K}}$ consists of the calibration uncertainty (increasing from $1 \%$ at $10^{-3} \mathrm{~Pa}$ to $2 \%$ at
$7.3 \times 10^{-7} \mathrm{~Pa}$ ) and of an assumed long-term instability of $3 \%$ over a six-month period of the reference gauge.

The PTB molecular beam expansion apparatus is shown in Fig. 1. The distance between the two orifices is $100 \mathrm{~mm}$, the diameter of the Knudsen cell orifice $2 \mathrm{~mm}\left(0.36 \mathrm{l} / \mathrm{s}\right.$ for $\left.\mathrm{N}_{2}\right)$ and of the calibration chamber $8 \mathrm{~mm}(5.8 \mathrm{l} / \mathrm{s})$. During calibration the system is pumped by a condensation cryopump, a titanium sublimation pump, and an ion pump. An additional turbopump is used only for initial evacuation or bake out, which is usually carried out at $350^{\circ} \mathrm{C}$.

The residual pressure of the calibration chamber is mainly determined by the outgassing of the gauge itself. Due to the conductance of $5.8 \mathrm{l} / \mathrm{s}$ the outgassing rate must be $5.8 \times 10^{-9} \mathrm{~Pa} l / \mathrm{s}$ to reach a residual pressure of $10^{-9} \mathrm{~Pa}$. This is a very low outgassing rate reached only by a few gauges. Outgassing rates of usual gauges range from $10^{-9} \mathrm{~Pa} l / \mathrm{s}$ up to $10^{-6} \mathrm{~Pa} l / \mathrm{s}^{2,3)}$

The sensitivity of an ion gauge is defined as 


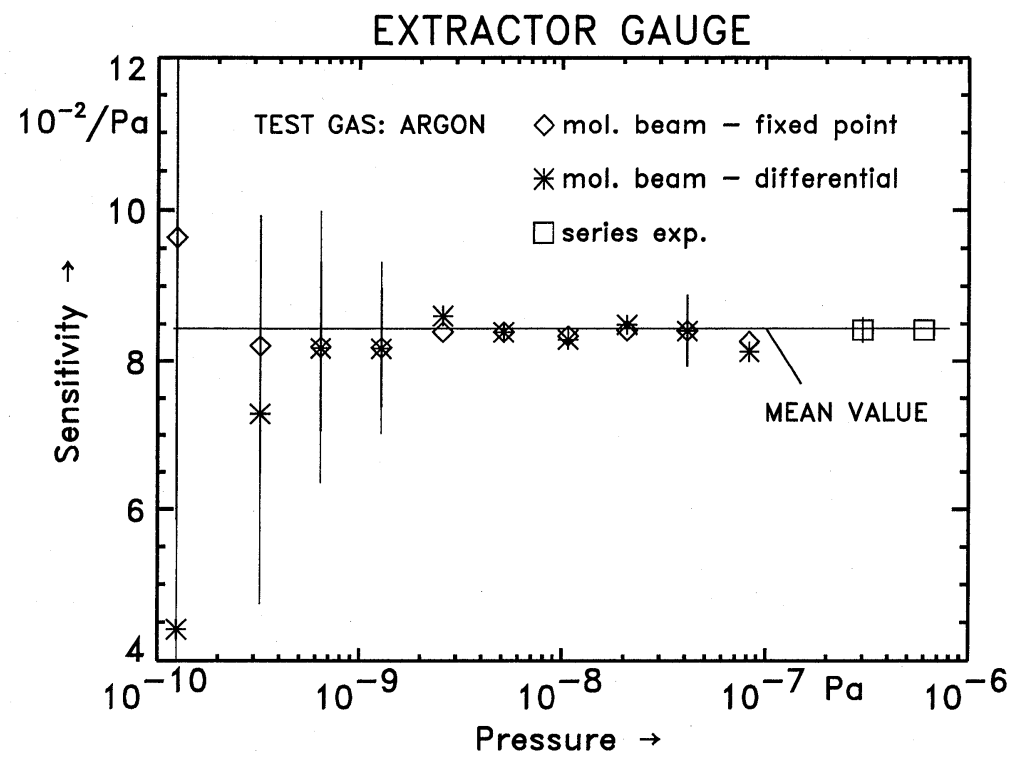

Fig. 2 Calibration of the sensitivity of an extractor gauge for argon from $10^{-10} \mathrm{~Pa}$ to $10^{-7} \mathrm{~Pa}$ with the molecular beam system and with a series expansion system. Typical uncertainty bars are shown (thick for fixed point method, thin for differential method).

$$
S=\frac{\Delta I_{\mathrm{c}}}{I_{\mathrm{e}} \Delta p_{\mathrm{cal}}}
$$

where $I_{\mathrm{e}}$ is the emission current and $\Delta I_{\mathrm{c}}$ is the change of collector current due to a change of calibration pressure $\Delta p_{\text {cal }}$. According to this definition the sensitivity may be measured in two ways.

1. The change of collector current $\Delta I_{\mathrm{c}}$ is measured with respect to a fixed value, usually $I_{\mathrm{c}}=I_{\mathrm{R}}$, with the residual current reading at $p_{\text {cal }}=0$.

2. The change of collector current between two neighboring calibration pressures, where $p_{\text {cal }}$ was changed by $\Delta p_{\text {cal }}$, is measured.

The first method will be called the fixed-point method hereafter, and the second, the differential method. Which one is better depends on the actual calibration. If the residual current has been determined incorrectly, the differential method will give better results; if $I_{\mathrm{R}}$ changes during the calibration sequence, the fixed-point method will give better results (in the case of the differential method the fluctuations will affect the smaller difference $\Delta I_{\mathrm{c}}$ between two points). In most cases the standard deviations of the fixed point method are smaller.

The residual current $I_{\mathrm{R}}$ of a gauge, which in our molecular beam system is mainly determined by the outgassing of the gauge, can be determined in two ways:

1. by measuring $I_{\mathrm{R}}$ over a longer period of time (30 $\mathrm{min})$, because $I_{\mathrm{R}}$ usually has a considerable long-term drift;

2. if the collector current $I_{\mathrm{c}}$ is measured against the collector current $I_{1}$ of the reference gauge in the Knudsen cell, $I_{\mathrm{R}}$ can be determined by extrapolating it to $I_{1}=0$. Since $I_{1}$ is high with respect to the residual current, even at the lowest calibration pressures, $I_{1}$ is not affected by its residual signal.

The uncertainty of a calibration above the residual pressure is mainly determined by the uncertainty of the calibration pressure. Below, however, the main uncertainty is due to the measurement of $\Delta I_{\mathrm{c}}$ and $I_{\mathrm{R}}$.

An example of the measurement of the sensitivity $S$ of an extractor gauge for argon is shown in Fig. 2. Before calibration the extractor was tested to have an X-ray-limit of $2 \times 10^{-10} \mathrm{~Pa}$ nitrogen equivalent. The ion current was measured with a high-quality digital picoammeter, read out by a computer. Twenty measured values were averaged. The asterisks indicate the result of the differential evaluation of the data, the diamonds, those of the fixed point method. The residual pressure was $8 \times$ $10^{-10} \mathrm{~Pa}$ (argon equivalent).

The calibration of the sensitivity at a series expansion primary standard at pressures above $10^{-7}$ $\mathrm{Pa}$ gave the result $S=8.42 \times 10^{-2} \mathrm{~Pa}^{-1}$. It can be concluded that even at calibration pressures smaller than residual pressure in the calibration chamber reasonable results can be obtained. 


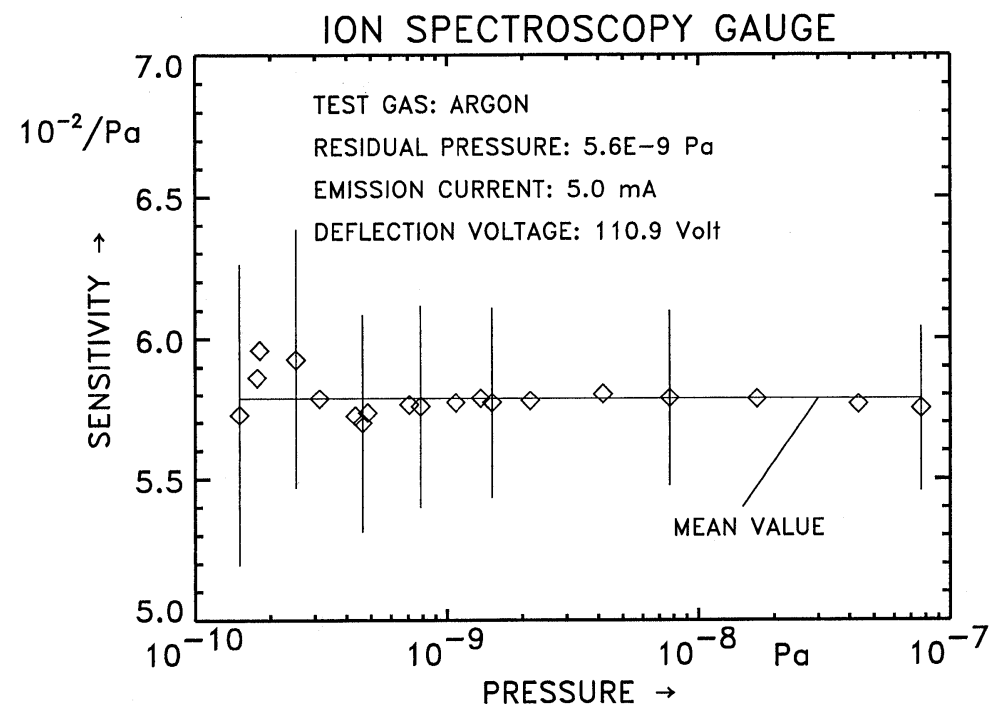

Fig. 3 Calibration of the sensitivity of an ion spectroscopy gauge for argon from $10^{-10} \mathrm{~Pa}$ to $10^{-6} \mathrm{~Pa}$ with the molecular beam system. Typical uncertainty bars are shown.

Another calibration is shown in Fig. 3 for the ion spectroscopy gauge of Watanabe. ${ }^{4)}$ The X-ray-limit was measured as $<7 \times 10^{-11} \mathrm{~Pa}$ (argon equivalent). Before calibration the residual pressure was $5.6 \times$ $10^{-9} \mathrm{~Pa}$. The residual reading $I_{\mathrm{R}}$, however, was stable enough to calibrate the gauge for calibration pressures considerably smaller than residual pressure and with less uncertainty than for the extractor gauge. We found relative large changes of up to $10 \%$ in sensitivity from day to day for this gauge.

The main advantage of the molecular beam method is that calibrations can be performed faster and with less expense than with the continuous expansion in the same pressure range, as will be described in the following. There are, however, also two major drawbacks: one must rely on a (relatively unstable) calibrated ionization gauge as the secondary standard, and the residual pressure is limited by the outgassing rate of the test gauge due to the small orifice of the calibration chamber. Therefore, PTB attempts to extend the lower limit of the calibration pressure generated with the continuous expansion method.

\section{Calibration in the UHV and XHV Ranges with a Continuous Expansion Standard}

The principle of the continuous expansion standards is to inject a gas flow of well-known rate $q_{\mathrm{pv}}$, generated by a flow meter, into a calibration chamber with a pump orifice of well-known conductance $C$. In the stationary equilibrium the injected gas flow is pumped through this orifice and, assuming the isothermal case with no backstreaming through the pump orifice, the calibration pressure is given by

$$
p_{\mathrm{cal}}=\frac{q_{\mathrm{pv}}}{C},
$$

In most cases the lower limit of the calibration pressure in a continuous expansion standard is determined by the lowest flow rate $q_{\mathrm{pv}}$ of about $10^{-5} \mathrm{~Pa}$ $l / \mathrm{s}$ (measured at room temperature) achievable with flow meters. Lower flow rates can be generated (see ref. $\left.{ }^{5}\right)$, although not with a fundamental measurement of the conductance in the flow meter, so that higher uncertainties and instabilities must be considered. With $q_{\mathrm{pV}}=10^{-5} \mathrm{~Pa} l / \mathrm{s}$ as the lower limit and a conductance of $100 \mathrm{l} / \mathrm{s}$ in the calibration chamber, one calculates $10^{-7} \mathrm{~Pa}$ as the lowest calibration pressure. The conductance of $100 \mathrm{l} / \mathrm{s}$ is already of relatively large size, which is only reasonably applicable with cryopumps.

The extend this lower limit without losing too much accuracy, a flow divider may be used. This will be realized in the new continuous expansion standard, under construction at PTB. The principal scheme of this system is shown in Fig. 4.

This calibration system consists of two chambers, an UHV vessel $V_{1}$ (base pressure $10^{-9} \mathrm{~Pa}$ ) and an XHV vessel $V_{2}$ (planned base pressure $10^{-11} \mathrm{~Pa}$ ), separated by a flow divider. To date, $V_{1}$ and the flow divider including the pump system have been operated and evaluated, while $V_{2}$ is under construc- 


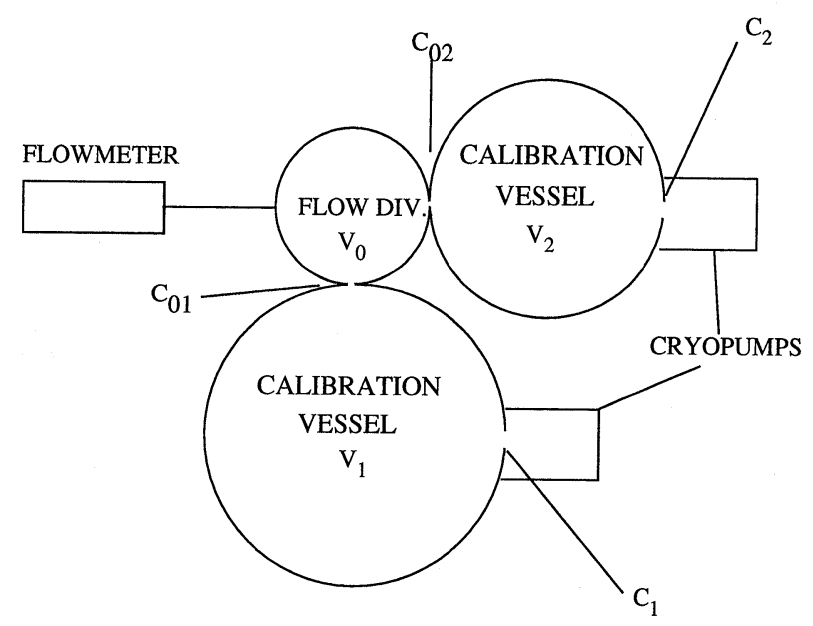

Fig. 4 Principal setup of the new continuous expansion standard at PTB. The flow divider $V_{0}$ divides the flow from the flow meter, with $99 \%$ going into the UHV calibration vessel $V_{1}$ and $1 \%$ into the XHV calibration vessel $V_{2}$.

tion. When both vessels are being pumped, the flow divider injects $99 \%$ of the gas flow into $V_{1}$ and $1 \%$ into $V_{2}$ through two differently sized orifices. Since the conductances of the pump orifices are the same (about $100 \mathrm{l} / \mathrm{s}$ for nitrogen), the calibration pressure in $V_{2}$ will be two orders of magnitude lower than in $V_{1}$. Both vessels are pumped with cryopumps. The cryopump in $V_{2}$, however, will be supported by a NEG pump and a titanium sublimation pump, both in parallel to the cryopump behind the pump orifice. With the above limit of $q_{\mathrm{pv}}$ $=10^{-5} \mathrm{~Pa} l / \mathrm{s}$, the lower limit of calibration pressure in $V_{1}$ is $10^{-7} \mathrm{~Pa}$ and $10^{-9} \mathrm{~Pa}$ in $V_{2}$ with about the same expected uncertainties $\left(3 \%\right.$ in $V_{1}$ and $3.5 \%$ in $V_{2}$ ).

It is planned that the calibration pressure in $V_{2}$ may even be extended to $10^{-11} \mathrm{~Pa}$ by applying lower flow rates, either by operating the flow meter in the constant conductance mode ${ }^{5}$ or by injecting the gas from a static expansion system.

To achieve a base pressure of $10^{-11} \mathrm{~Pa}$ in $V_{2}$ an outgassing rate (mainly hydrogen) of $10^{-12} \mathrm{~Pa} / / \mathrm{s}$ $\mathrm{cm}^{2}$ from the walls must be attained. For this reason vessel $V_{2}$ will be produced of vacuum-fired $316 \mathrm{LN}$ stainless steel. $V_{1}$ was produced in the same manner to test the outgassing rate of the material. After moderate baking at $250^{\circ} \mathrm{C}$ an outgassing rate of $2 \times 10^{-12} \mathrm{~Pa} / / \mathrm{s} \mathrm{cm}^{2}$ (nitrogen equivalent) was achieved in $V_{1}$, measured with the pressure rise method by a spinning rotor gauge (SRG). Since there are doubts as to whether the outgassing rate may depend on the applied pump speed, ${ }^{1)}$ we have carried out an additional experiment to clarify this question, which will be reported in the next sec- tion.

It is planned that the evaluation of this new calibration system at PTB will be completed in 1995.

\section{Measuring of Outgassing Rates}

Two methods for measuring outgassing rates are commonly used: the pressure rise method in an isolated volume and the throughput method in a pumped volume. In both methods it is assumed that the outgassing rate is not affected by readsorption. Other-wise, the outgassing rate would depend on the pump speed applied to the vessel where outgassing rate is measured. Malev published a theory in $1973^{1)}$ which predicted just this dependence of the outgassing rate on applied pump speed even for usual experimental conditions.

To solve the diffusion equation, two boundary conditions are commonly assumed: the concentration of $\mathrm{H}$ atoms at the surface exposed to atmosphere is in equilibrium to its atmospheric concentration, and at the surface exposed to vacuum, equal to zero (all atoms are immediately desorbed). The latter boundary condition was replaced in Malev's theory by an equation describing the stationary equilibrium at the surface and in the volume. A result of his theory is that the outgassing rate depends on the square root of pump speed.

If Malev's theory is true, this would mean that the higher the applied pumping speed the higher the outgassing rate would be and the expected ultimate pressures would not be reached.

To check the validity of this theory, we developed a new method of outgassing rate measure- 


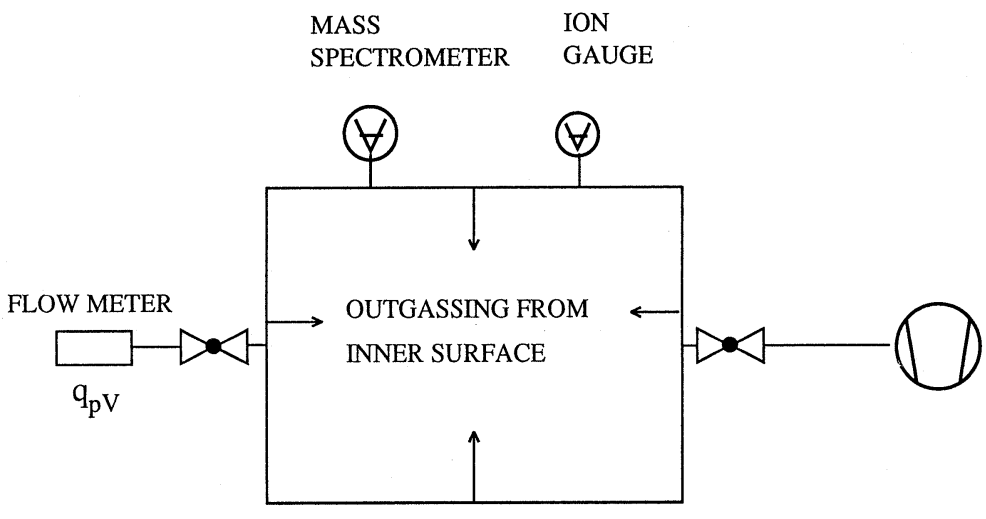

Fig. 5 Experimental setup for measuring outgassing rates with a flow meter. The gas flow from the flow meter is set equal to the total outgassing rate. The ultimate pressure of the vacuum system is eliminated by measuring at different pump speeds changed with a variable valve.

ment, where the outgassing rate is measured in dependence of pumping speed. We compared the results of this method with the classical methods of outgassing rate measurement.

Figure 5 shows the experimental setup for the method, which will be called, in the following, the "flow meter method". Before gas is injected into the chamber, the residual reading $p_{0}$ of the calibrated ion gauge is taken at an arbitrary position of the variable right-angle valve. Then a well-known gas flow $q_{\mathrm{fm}}$ from the flow meter (hydrogen) of about the same size as the total outgassing rate is injected into the chamber. With $p_{1}$ read from the calibrated gauge, the effective pumping speed is obtained by

$$
S_{\text {eff }}=\frac{q_{\mathrm{fm}}}{p_{1}-p_{0}}
$$

To enhance the accuracy, this can be repeated for several $q_{\mathrm{fm}}$. It is also repeated for various $S_{\text {eff }}$. The residual reading $p_{0}$ is given by

$$
p_{0}=p_{\text {pump }}+\frac{q_{\text {out }}}{S_{\text {eff }}},
$$

where $p_{\text {pump }}$ is the ultimate pressure of the vacuum system downstream of the valve, and $q_{\text {out }}$ the total outgassing rate upstream, which is assumed to be independent of $S_{\text {eff }}$ in contrary to Malev's theory. By fitting the experimental data to equation (5), both $p_{\text {pump }}$ and $q_{\text {out }}$ can be obtained. The pumping speed of the ion gauge does not affect the result, since it is included in $S_{\text {eff }}$.

The uncertainty of this method is determined by the accuracy of $q_{\mathrm{fm}}$ (approximately $4 \%$ at gas flows typical for UHV outgassing), the uncertainty of the calibrated ion gauge (5\%), and statistical uncertainties (typically $5 \%$ ), and is in total, $15 \%$, which is a low uncertainty compared to the throughput method. For $S_{\text {eff }}<1 l / \mathrm{s}$ the times to attain a stable equilibrium for measurement increase considerably, and the uncertainty of $S_{\text {eff }}$ may rise to about $50 \%$ at $S_{\text {eff }}=10^{-2} \mathrm{l} / \mathrm{s}$.

The method described above was applied to measure the outgassing rate of a vacuum-fired vessel $V_{1}$, described in the preceding section, and the non-vacuum-fired connection tube to the flow meter. Attached to the chamber were two extractor gauges, one of them calibrated, a spinning rotor gauge (SRG), a quadrupole mass spectrometer (QMS), a leak valve, and a right-angle valve (DN 63). Downstream, immediately neighboring the leak valve, a BA-gauge was mounted. Total volume was $29.7 l$ and surface area $8740 \mathrm{~cm}^{2}$. The chamber was pumped with two magnetically suspended turbomolecular pumps in series, rough pumped with a dry pump.

Unfortunately, it was found, that the outgassing rate of the operating QMS was more than one order of magnitude larger than the total outgassing rate of the chamber including the ion gauges, so that the QMS was not used. Even in the nonoperating mode we suspect that the outgassing rate from the QMS was considerable.

The chamber was baked for $72 \mathrm{~h}$ at $250^{\circ} \mathrm{C}$. After pumping down to about $4 \times 10^{-8} \mathrm{~Pa}$ (hydrogen equivalent) ultimate pressure, the outgassing rate with ion gauges off was measured by the pressure rise method with the SRG to be $2 \times 10^{-7} \mathrm{Pal} / \mathrm{s}$ (hydrogen equivalent and at $23^{\circ} \mathrm{C}$ ). The pressure rise was linear over a 4-week period.

Subsequently, the outgassing rate was measured by the flow meter method on two consecutive days. The fit (line 1) according to equation (5) and the 


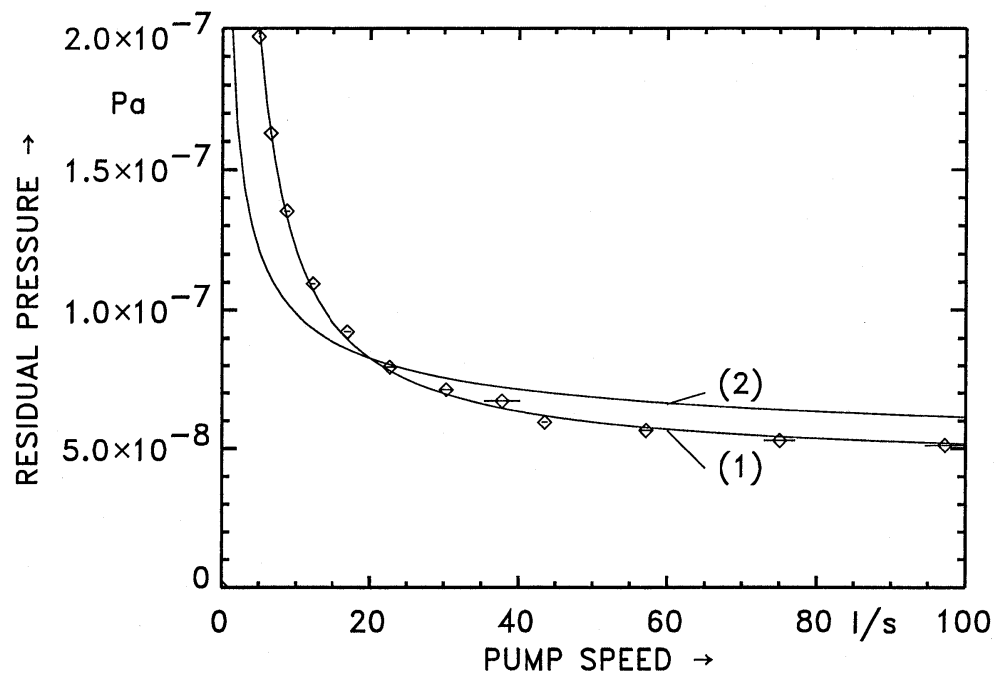

Fig. 6 Residual pressure versus pump speed. The fit (1) according to equation (5) yields an ultimate pressure of the vacuum system $p_{0}=4.4 \times 10^{-8} \mathrm{~Pa}$ and an outgassing rate of $q_{\text {out }}=7.73 \times 10^{-7} \mathrm{~Pa} l / \mathrm{s}$. The fit curve (2) would be expected, if $q_{\text {out }}$ depended on the square root of pump speed (Curves (1) and (2) set equal at $20 \mathrm{l} / \mathrm{s}$ ).

measured data of the second day are shown in Fig. 6. From the fitted curve we obtained $p_{\text {pump }}=4.4 \times$ $10^{-8} \mathrm{~Pa}$ and $q_{\text {out }}=7.7 \times 10^{-7} \mathrm{~Pa} l / \mathrm{s}$, both values in hydrogen equivalent and measured at $23^{\circ} \mathrm{C}$. On the first day, the results were $p_{\text {pump }}=4.8 \times 10^{-8} \mathrm{~Pa}$ and $q_{\text {out }}=6.9 \times 10^{-7} \mathrm{~Pa} l / \mathrm{s}$. It was plausible that $q_{\text {out }}$ was higher on the second day, because at the end of the first day the chamber was filled by mistake with $1 \mathrm{kPa}$ hydrogen for a short time. The difference of $q_{\text {out }}$ of about $5 \times 10^{-7} \mathrm{~Pa} l / \mathrm{s}$ between the pressure rise and flow meter methods may be explained by the outgassing rate of the extractor gauge and is a rather typical value reported also from other experimenters. ${ }^{3)}$

The results, shown in Fig. 6, are fully compatible with the assumption that the outgassing rate is independent of pumping speed from $6 \mathrm{l} / \mathrm{s}$ up to 100 $\mathrm{l} / \mathrm{s}$. If it did depend on the square root of pumping speed according to Malev's theory, the characteristic of line (2), which was set equal to the fit curve at $20 \mathrm{l} / \mathrm{s}$, would have been measured. We must note, however, that only about $30 \%$ of the total outgassing rate was caused by hydrogen outgassing from the walls, the remainder being caused by outgassing from the hot ionization gauge used for measurement, which may not depend on the square root of pumping speed since it is a different mechanism of outgassing (i.e., outgassing stimulated by electron bombardment, thermal radiation, chemical reactions, photon bombardment and so on).

The flow meter method was also compared to the throughput method. For this the large valve to the pumping system was closed and the chamber was pumped through the leak valve, over which the pressure difference was measured with the calibrated extractor gauge and BA-gauge. The effective pumping speed was determined as above. At $S_{\text {eff }}$ $=0.13 \mathrm{l} / \mathrm{s}$ we obtained $q_{\text {out }}=9.5 \times 10^{-7} \mathrm{~Pa} \mathrm{l} / \mathrm{s}$ (flow meter method) and $q_{\text {out }}=9.3 \times 10^{-7} \mathrm{~Pa} l / \mathrm{s}$ (throughput method), and at $S_{\text {eff }}=0.04 \mathrm{l} / \mathrm{s}, q_{\text {out }}$ $=1.8 \times 10^{-8} \mathrm{~Pa} l / \mathrm{s}$ (flow meter method) and $q_{\text {out }}$ $=1.7 \times 10^{-8} \mathrm{~Pa} l / \mathrm{s}$ (throughput method). The results were in agreement within the uncertainties of the two methods. As mentioned above, it took hours to get a fair equilibrium, and at $S_{\text {eff }}=0.04$ $l / \mathrm{s}$ there may be a systematic error in both cases. The higher outgassing rate was partly du to the operation of another hot ionization gauge.

The outgassing rate did not show a square root dependence on pumping speed, which calls Malev's theory into question. One of the main problems in Malev's theory may be the assumption of a stationary equilibrium. At high temperatures, the gas flow by diffusion is strongly time-dependent. The assumption of stationary equilibrium may therefore lead to an erroneous concentration profile at bakeout temperatures and, since this concentration profile is used under the boundary condition at low temperatures, also to an erroneous outgassing rate.

\section{Discussions and Conclusions}

It is possible to calibrate vacuum gauges down to 
$10^{-10} \mathrm{~Pa}$ with the molecular beam method at PTB. Two major drawbacks accompany this method. The residual pressure in the calibration chamber is determined by the gas release from the gauge itself and the calibration pressure is deduced from the reading of the relative unstable ionization gauge. Although it is possible to adjust calibration pressures to lower than residual pressure in the calibration chamber, it would be desirable to have calibration chamber where residual pressure is below the lowest calibration pressure.

For this reason, in the near future, calibrations in the XHV will be performed at PTB with a new continuous expansion standard. Due to the higher pumping speed applied to the calibration chamber during calibration the residual pressure will be lower than in the molecular beam system. The planned residual pressure of the calibration system is $10^{-11}$ $\mathrm{Pa}$. The necessary low flow rates will be generated by means of a flow divider.

Outgassing rates of the vacuum-fired stainless steel, which will be used in the new standard, are roughly compatible (within a factor of two) with the planned residual pressure.

To check whether the different outgassing measurement methods give the same results, we used the linear pressure rise method, the throughput method, and the new flow meter method for the same chamber. The results of the three method were all compatible with each other, if the outgassing of the gauges was considered, and by the new flow meter method, it was shown that there is no evidence that outgassing rate should depend on pumping speed as proposed by Malev.1)

\section{References}

1) M. D. Malev: Vacuum 23 (1973) 43.

2) F. Watanabe: J. Vac. Sci. Technol. A11 (1993) 432.

3) M. Saitoh, K. Shimura, T. Iwata, T. Momose and H. Ishimaru: J. Vac. Sci. Technol. A 11 (1993) 2816.

4) F. Watanabe: J. Vac. Sci. Technol. A 10 (1992) 3333.

5) K. Jousten, G. Messer and D. Wandrey: Vacuum 44 (1993) 135. 